\title{
Le marché et la commercialisation du gros bétail dans le Moyen-Soudan*
}

\author{
par G. JOURDAIN, M. DRAHON ef M. RÉVILLON
}

D'aucuns pensent et s'en vont répétant, après l'avoir lu ef relu dans les écrits compilés, que l'éleveur de ce pays, demeuré dans une boophilie stérilisante, ne commercialise pas son bétail. La vue de vieilles vaches ** suivant péniblement les troupeaux transhumants et demandant la mort aussi bien au temps qu'aux fauves, est pour beaucoup à l'origine de cette idée qu'il faut pourtant bien abandonner lorsque l'on fréquente les marchés à bétail coutumiers du MoyenSoudan.

Dans cette région les transactions commerciales du bétail ont trois buts principaux : l'approvisionnement du marché intérieur de la viande, la fourniture de bœufs de trait aux cultivateurs, l'exportation d'animaux de boucherie vers les pays dépourvus d'élevage du golfe du Bénin.

Les modalités de ces transactions sont diverses selon le but poursuivi et selon les régions. Dans une grande partie du Moyen-Soudan - pays Bambara, Malinké, Sénoufo - les transactions sont sporadiques et par conséquent ni spectaculaires ni contrôlables : il n'en est pas de même dans le cœur même du pays c'est-à-dire dans le Delta vif du Niger qui est géographiquement et économiquement favorable à l'existence de marchés importants.

La connaissance de ces circuits commerciaux

(*) Cet article a déjà paru dans le Bulletin de 'jaison de l'Office du Niger (nov.-déc. 1959 et janv.-févr. 1960).

(**) Les éleveurs conservent certaines viei!les vaches rescapées des grandes enzooties et par conséquent naturellement immunisées contre les principales maladies contagieuses: c'est grâce à ces bêtes qu'un troupeau décimé par une épizootie survenue pourrait être reconstitué. L'entretien de ces vaches correspond à une prime d'assurance ; cette sujétion est de moins en moins nécessaire, au fur et d̀ mesure que les méthodes pasteuriennes s'implantent et s'adaptent dans ce pays.

Rev. Elev. Méd. vél. Pays trop., 1960, 13, no 4. présente un intérêt certain pour les acheteurs de bétail, ainsi que pour la recherche d'une amélioration de la commercialisation.

La répartition géographique des marchés, leur interdépendance, leur zone d'action, ainsi que leurs vocations peuvent être figurées par la carte ci-jointe.

A première vue deux groupes de marchés se distinguent selon leurs vocations pour l'exportation vers le Ghana ou vers la Côte d'Ivoire ef Bamako. En approfondissant les choses ces deux groupes se distinguent sur bien d'autres points : c'est pourquoi nous les examinerons séparément. Pour la commoditć de l'exposé nous désignerons le premier groupe par le vocable Marchés Diawambé et le second par Marchés Bambara.

\section{I. - LES MARCHÉS DIAWAMBÉ}

Les marchés Diawambé sont répartis au cœur et de part ef d'autre du Delta vif du Niger. Les courants commerciaux polarisés par le Ghana empruntent deux chaînes de marchés :

- l'une (Gatié, Togueré, Kombé, Oualo, Ténenkou, Mopti) sur la rive gauche du Delta.

- I'autre (N'Gouma, Korientzé, Kona, Fatoma, Mopti) sur la rive droite du Delta.

L'existence de ces chaînes de marchés est favorisée par la nature des lieux. En effet l'important marécage que constitue le Delta vif du Niger recèle des pâturages de décrue et de saison sèche très importants et capables d'entretenir de janvier à juin un cheptel évalué à 500.000 têtes. Ces pâturages se nomment Bourgou et, pur contraction dans le langage des éleveurs, le Delta vif du Niger est désigné par le mot Bourgou. Le Bourgou sépare en période de crue et réunit en saison sèche, deux zones d'éle- 
vage que les éleveurs désignent par le mot Séno: le Séno du Sahel au nord, le Séno de Bandiagara au Sud. Lcs Sćno sont des pâturages de saison des pluies : c'est dire que l'ensemble Bourgou-Séno forme un biotope complet pour les troupequxqui savent transhumer.

Lès rives du Bourgou à proximité de bons pâturagcs où en toute saison le bétail peut se concentrer sont donc favorables à l'existence de marchés. La rive droite, d'autre part, est en relation constante avec le Ghana qui outre le bétail est acheteur, de poisson sec et fumé commercialisé en ces lieux (4.000 tonnes annuellement pour une valeur de 350 millions de francs c. f. a.).

Nous avons parlé dès l'abord d'interdépendance des marchés, de chaînes de marchés et de courant commercial polarisé vers le sud : cela nécessite une explication, et pour ce faire le mieux est de suivre les périgrinations d'un bœuf mis en vente pour l'exportation.

Supposons par exemple un bouf issu d'un ćlcvage de la région de Sarafere (un bœuf Guimbala). II sera naturellement présenté au marché de N'Gouma ; là, il peut être vendu à un exportateur, intégré à un troupeau d'exportation (100 têtcs cn moycnne) et partir pour le Ghana directement par Doventza, Ouaga. Tout aussi bien il peut ne pas avoir trouvé l'acquéreur convenable; dans ce cas, sans attendre le prochain marché de N'Gouma, il sera dirigé vers le marché de Korientze pour y tenter sa chance. S'il ne trouve pas acquéreur, il se présentera d̀ Kona puis à. Fatoma et Mopti (a), sera finalement

(a) Pendant l'inondation, d'octobre à janvièr, le marché à bétail de Mopti ne peut avoir lieu ; le marché de Fatoma le supplée alors.

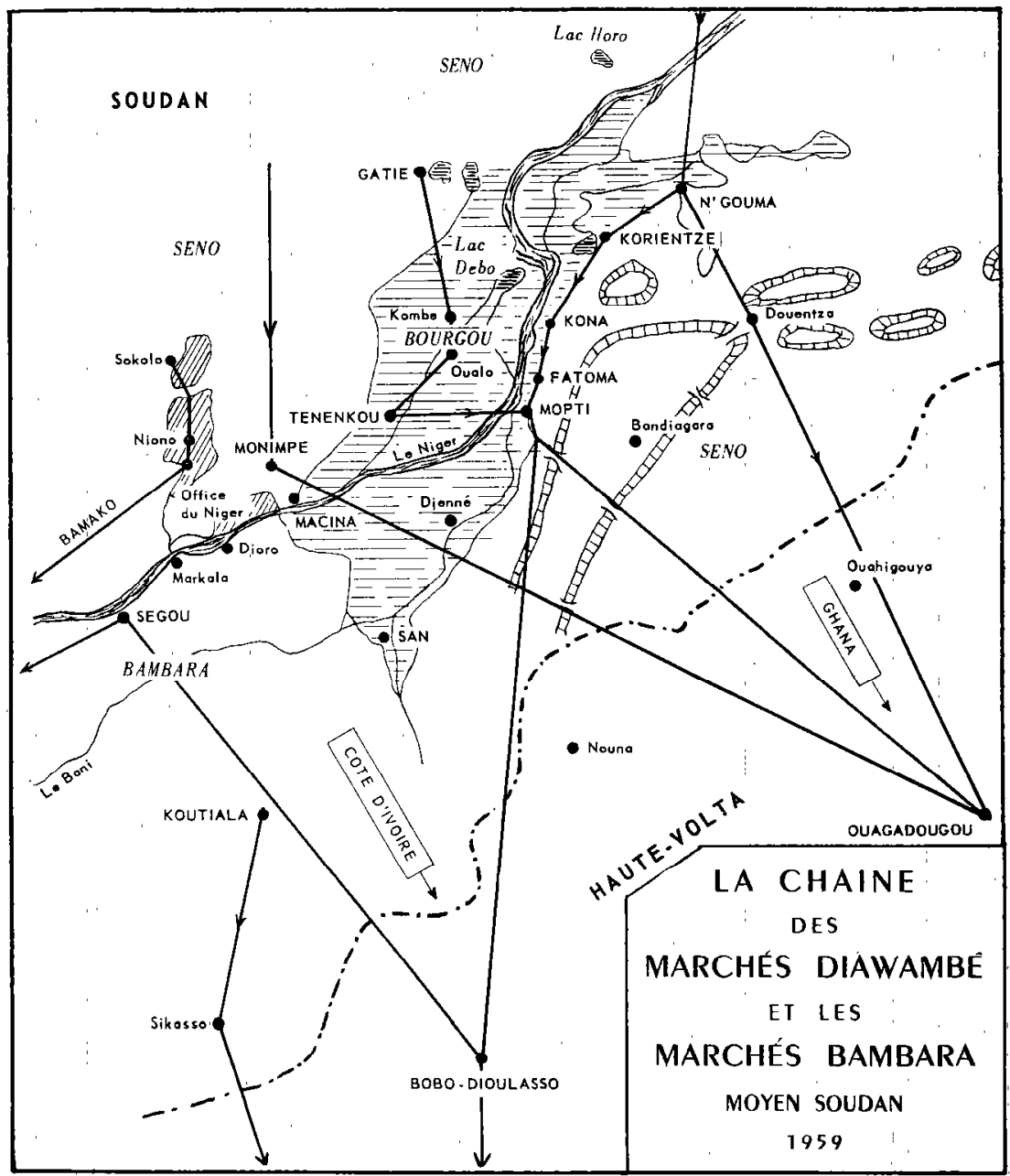


vendu, et partira intégré dans un troupeau d'exportation (un tiogal) définitivement constitué qui suivra la route Baboye-Ouaga-Tenkodogo.

De même un bœuf présenté à Gatie pourra être vendu à Tenenkou après s'être presenté à Toguère, Kombe et Oualo; il sera alors intégré à un tiogal qui sera exporté au Ghana par la route Mopti-Baboye-Ouaga-Tenkodogo.

En parcourant ces chaînes de marchés, un bœuf peut être marchandé à Kombe par exemple puis à Oualo et être acheté par le même amateur à Tenenkou seulement, c'est-à-dire que les acheteurs peuvent suivre le même périple. D'autre part un bœuf peut, dans les marchés de la rive droite, passer par plusieurs mains avant d'être acquis par l'exportateur définitif; c'est dire que ces marchés sont jalonnés d'intermédiaires.

L'organisation en chaîne des marchés Diawambé apparaît donc ainsi. II reste à ajouter que les jours fixés pour chacun d'eux le sont de telle sorte que marchands ef bestiaux ont le temps, sans toutefois en perdre, de passer d'un marché à un autre, compte tenu des distances ef des difficultés qui séparent les lieux. Ces marchés sont hebdomadaires :

\section{Marchés rive gauche}

De janvier à juillet :

$\begin{array}{cccc}\text { Gatie } & \text { Kombe } & \text { Oualo } & \text { Tenenkou } \\ \text { retour de } & - & - & - \\ \text { transhumance } & \text { Samedi } & \text { Lundi } & \text { Jeudi }\end{array}$

\section{Marchés rive droite}

Toute l'année sauf Mopti :

N'Gouma Korientze Kona Fatoma Mopti Jeudi Lundi Mercredi Mardi Jeudi

II reste aussi, sans aller plus avant, à chiffrer l'importance de ces chaînes. Cela est assez malaisé du fait que le décompte des bêtes sur chaque marché (ce qui est faisable et fait depuis plusieurs années) donne un chiffre brut ne tenant pas compte des interdépendances ef ne correspondant que de loin au nombre des animaux commercialement évacués de la région. Cependant il est intéressant de noter ces chiffres bruts, en se réservant de les interpréter par lo suite.
En moyenne il est dénombré annuellement: sur les marchés de la rive gauche 20.000 bovins et 60.000 sur les marchés de la rive droiłe.

Ce n'est pas rien; cela peut même étonner certains journalistes omniscients. Nous achèverons cet alinéa en livrant les chiffres suivants relatifs aux marchés du Séno de Bandiagara : Doventza: 10.000 têtes, Bankass-Ouenkono: 10.000 têtes.

Pour acquérir une certaine connaissance de ces marchés nous nous proposon's, après avoir croqué les chalands qui les fréquentent (les acheteurs, les vendeurs et les courtiers) de décrire le bétail qui s'y présente qualitativement et quantitativement.

A) Les vendeurs (Agriculteurs, Maures, Peulhs Touaregs).

1. Les Agriculteurs. II n'y a pas si longtemps, seuls certains groupes ethniques étaient spécialisés dans la culture, exerçant leur art pour leur propre comple (Bambara par exemple) ou pour le compte de leurs maîtres (Rimaïbé pour les Peulhs, etc...). D'autre part, les colons de l'Office du Niger recrutés en pays Mossi et dans la région de Tougan étaient aussi des agriculteurs exclusifs. Ces distinctions ethniques attachées a une spécialité deviennent inutiles car, avec l'utilisation de la charrue, tout cultivateur est amené à s'intéresser à l'élevage et par rapport à cette activité leurs habitudes deviennent communes et se confondent à celles des éleveurs d'origine, qui aujourd'hui s'intéressent à l'agriculture.

Les agriculteurs vendent surtout des bœufs de labour réformés. Quand ils ont besoin d'argent pour l'impôt, le mariage, ou pour acquérir un nouvel attelage, ils consentent, avec autant de réticence que leurs congénèreś éleveurs, à vendre des taurillons, des génisses même, mais jcmais de vaches en rapport. Les agriculteurs se rencontrent sur tous les marchés cités dans celle éfude.

2. Les Moures fréquentent quelquefois le marché de Tenenkou mais surtout la chaîne de marchés Sokolo-Niono, dont nous parlerons plus loin. Les Maures vendent pour acheter du grain qu'ils vont commercialiser en Mauritanie où les cultures vivrières sont insuffisantes; c'est dire que nous les voyons fréquenter les marchés au moment des récoltes et pendant la saison sèche. Pour ce trafic les Maures font une grande con- 
sommation de bœufs-porteurs ; c'est pourquoi ils ont tendance à conserver les taurillons. Ils vendent des génisses de 2 ans et surtout des bœufs-porteurs réformés : disons tout de suite que ces bœufs sont impropres à l'exportation vers le sud car ils sont affaiblis et supportent très mal l'humidité.

3. Les Peulhs fréquentent les marchés Diawambé toute l'année. A l'heure actuelle au moins 50 p. 100 d'entre eux s'intéressent aussi à l'agriculture ; cependant les produits de l'élevage demeurent leur ressource principale. Leurs besoins sont relativement importants et ont tendance à augmenter car ils aiment les signes extérieurs de richesse. Leurs femmes sont coquettes, et ils apprécient plus qu'on ne pense le confort apporté par notre civilisation ; aussi exploitentils leurs troupeaux assez correctement. Mis à 'part le commerce du lait, les Peulhs font de l'élevage pour produire des bœufs d'exportation. Ils vendent aussi des vaches stériles (les Rimaré) qui sont excellentes pour l'cxportation, et les vieilles vaches réformées. De plus, depuis l'avènement de la charrue ils commercialisent les taurillons et les jeunes bœufs ainsi que les bœufs de labour réformés. Les vaches en rapport ne sont jamais vendues et les génisses très rarement.

4. Les Touaregs fréquentent les marchés Diawambé de la rive gauche ainsi que les marchés nord de la rive droite. Les Touaregs, exclusivement éleveurs, vivent exclusivement de leurs troupeaux (ils ne vendent pas le lait). Ils doivent tout acheter (grains, habillement) et sont donc poussés à commercialiser assez intensément leur bétail : bœufs d'exportation, jeunes bœufs, taurillons, génisses de plus d'un an, vaches stériles, vieilles vaches réformées. Les vaches en rapport ne sont pas mises en vente.

B) Les acheteurs. Le bétail est le livret de caisse d'épargne des éleveurs, ainsi d'ailleurs que des agriculteurs de cette région. Pour eux en effet, it est imprudent de conserver le numéraire par devers soi, car il est bien difficile de le mettre en sécurité dans des concessions mal protégées, qui sont souvent la proie des incendies, il est d'autre part bien malaisé de ne pas le laisser dilapider par les proches. C'est pourquoi agriculteurs et éleveurs convertissent toute somme disponible en bétail. Sur les marchés, après leurs ventes et leurs achats, nous les voyons donc, quand ils le peuvent, acheter de jeunes animaux qu'ils incorporent à leurs troupeaux: génisses de préférence sinon un taurillon (une génisse vaut deux taurillons au moins).

Les Agriculteurs sont acheteurs de bœufs de travail dont le commerce lié au développement de la charrue, va croissant. La demande porte sur des animaux de 3 à 4 ans suffisamment développés el assez jeunes pour pouvoir être dressés à la charrue : c'est une demande assez nouvelle (deux décades) dont l'influence sur la physionomie des marchés est tout à fait notable ef ira croissant. Les Peulhs sont acheteurs au mêmetitre que les agriculteurs. Les Maures achètent des vieilles vaches réformées, car certains d'entre eux sont spécialisés dans l'exportation de cette catégorie d'animaux. Les Touaregs ne sont pas acheteurs.

C) Les bouchers. Cette profession est peu développée en quantité comme en qualité : elle n'est viable que dans les centres et les marchés importants où les consommateurs sont en nombre suffisant. Quoi qu'il en soit, dans cette région où le poisson se trouve en grande quantité, la viande n'est pas très recherchée. Cependant les marchés Diawambé sont fréquentés par une trentaine de bouchers, acheteurs annuellement d'environ 2.000 bovins impropres à l'exportation : vieilles vaches, bœufs de labour et bœufs-porteurs réformés.

D) Les exportateurs. Toute personne suffisamment riche peut spéculer sur le bétail d'exportation : l'affaire peut être mauvaise ou très bonne (la culbute est possible) suivant les fluctuations du marché de la viande au Ghana où l'offre et la demande subissent dès à-coups importants. Mais pour ce faire il est pratiquement indispensable de passer par l'intermédiaire d'individus qui monopolisent ce trafic: il est quasi impossible à quiconque 'ne fait pas partie de certaines confréries, d'acheter du bétail d'exportation, de le convoyer ef de le vendre au Ghand par ses propres moyens. Deux groupes de marchands sont spécialisés dans ce trafic: les Diakanké ef les Diawambé.

Les Diakanké sont des Marka implantés dans la région du Diaka. Eleveurs et traditionnellement commerçants, ils constituent chaque année des troupeaux d'exportation en puisant dans leur 
propre élevage, en achetant sur les marchés et en prenant en charge le bétail d'autres éleveurs de la région. Les Diakanké exportent sur Bamako (marché de Kati-Kambila) ef sur le Ghana où ils ont les mains libres pour commercer et où ils jouissent d'ailleurs d'une très bonne réputation.

Les Diawambé forment un groupe ethnique très caractéristique dispersé en réseau dans dans toute l'Afrique Occidentale. lls sont issus de Peulhs et de Marka : ils ont hérité des Marka le sens du commerce et des Peulhs la connaissance du bétail. Ils sont les courtiers des Peulhs et connus dans ce rôle bien avantl'époque de Cheikou Amadou.

S'agissant de bétail leur courtage porte non seulement sur l'exportation mais aussi sur toute transaction sur les marchés.

Pour l'exportation, les Diawambé du Macina (les Koïta, les Bocoum, les Tiokari, les Landouré) sont en «cheville» avec les Diawambé du Ghana : ils forment ainsi une organisation audessus de laquelle il est bien difficile de passer (la chose a été tentée bien des fois sans succès par des Ghanéens et autres étrangers). Il est vrai d'ailleurs, qu'une telle organisation est nécessaire pour vaincre les embûches inimaginables attachées à ce trafic. Les Diawambé achètent et exportent à leur compte, mais surtout exportent pour le compte de tous spéculateurs ayant lové leurs services.

E) Les courtiers. Sur les marchés du Bourgou toute transaction de bétail est contrôlée ou effectuée par un courtier Diawambé que l'on nomme Teyfado. Tout vendeur, tout acheteur de bétail utilise les services d'un Teyfado.

La veille du marché, le vendeur présente et confie au Teyfodo l'animal qu'il veut vendre en lui indiquant le prix qu'il désire en retirer. Si la vente est réalisée le vendeur donne une commission au courtier (200 fr par bovin actuellement).

L'acheteur marchande avec le Teyfodo; si l'accord se fait il lui paye la somme convenue et également une commission. Si le prix de la vente est supérieur à celui demandé par le vendeur, celui-ci en bénéficie mais augmente la commission du courtier.

A première vue ce courtage peut sembler inutile ou abusif ; cela n'est pas si vrai cor, pendant le courtage, le Teyfado endosse certaines respon- sabilités qu'il serait pour bien des raisons difficile aux chalands de supporter : l'animal confié au Diawambé par le vendeur est dès lors placé sous la responsabilité de ce dernier, le parcage. l'abreuvement, l'entretien, la surveillance lui incombent. De même l'animal vendu reste pendant deux jours après la vente sous la responsabilité du Teyfado.

Ce système apporte aux chalands des avantages certains : il les dégage du souci d'entretenir et de surveiller leurs animaux dans un village mal connu d'eux. D'autre part. il les garantit du vol, de la fuite, de la perte (le Diawambé saura toujours retrouver un animal échappé et réfugié dans les troupeaux voisins, qu'il connaît bien); de plus, dans une certaine mesure, l'acheteur dispose d'un certain recours si un vice caché se révèle dans les deux jours qui suivent la vente.

Ainsi les marchés à bétail du Bourgou sont-ils entièrement contrôlés par les Diawambé : il semble même que ce soit eux qui les ont créés ; on peut estimer à une centaine le nombre des courtiers en exercice.

F) Le bétail présenté. La présentation du bétail sur les marchés n'est pas faite sans méthode, ni sans astuces. En général chaque Teyfado groupe les animaux d'exportation et les jeunes bœufs. $1 /$ présente à part les vaches, les génisses et les taurillons. Le marchand recherche la place favorable pouvant mettre en valeur son bétail : ainsi les bovins longilignes sont placés dans un basfond ou dans la mare afin de leur couper les pattes et de les faire paraitre plus ronds; les brévilignes sont massés sur les buttes pour augmenter leur prestance...

Nous allons passer en revue les catégories de bovins mis en vente, en débutant (noblesse oblige) par le bœuf d'exportation.

Hormis la sonté bien entendu, le bœuf d'exportation doit posséder deux qualités essentielles: l'aptitude à effectuer journellement trente bons kilomètres tout en s'alimentant et, du même coup, l'aptitude à traverser rivières et marécages.

La qualité des membres prime la qualité de la viande, car il s'agit d'effectuer en trente jours le millier de kilomètres semés d'embûches qui sépare le Bourgou du Ghana ; arrivés à Bakwu en ayant perdu un minimum de poids, les bœufs doivent posséder assez de réserves pour pouvoir se disperser dans les villages du Ghana. 
Ces qualités essentielles sont naturellement acquises par le bétail produit en élevage extensif au cours des continuelles transhumances. Les qualités requises atteignent leur meilleur développement chez les boufs âgés d'au moins 8 ans ayant transhumé régulièrement chaque année dans les bourgoutières du delta vif du Niger.

A poids égal, l'âge requis et l'aptitude à la marche étant acquis, la valeur du bouf d'exportation varie suivant son aptitude à franchir les cours d'eau, les marécages et à résister à l'humidité. Cette aptitude est attachée à l'origine du bétail reconnaissable à la conformation, la robe, le cornage ; aussi devons-nous classer les divers types de zébus rencontrés sur les marchés.

\section{1) Du SENO-NORD nous viennent:}

- les Tiapatidji : Bceufs maures hydrophobes et par conséquent difficilement exportables vers le Ghana. Vont sur Bamako.

- les Haoussa : Boufs venant de la région de Niafunké peu habitués à l'eau. Cependant exportables sur le Ghana.

- les Bouari : Zébus peulhs, Warbé de Nampala, du Kareri et du Kouroumari, suffisamment habitués à l'eau.

- les Tionadji : Zébus peulhs du Farimaké bien habitués à l'eau.

- les Bourgamedji : Bœufs Touaregs de la rive Haoussa très habitués aux marais longeant le fleuve.

\section{2) DU BOURGOU nous viennent :}

- les Gimbalakodji : Bœufs peulhs de la zone nord-est du Debo.

- les Bourgoukodji : Bœufs peulhs du Macina.

\section{3) Des SENO SUD-EST nous viennent:}

- les Foulankriadji : Bœufs élevés dans la région de Korarou-Korientzé-Konza. Boufs peulhs touaregs bien habitués à l'eau.

- les Hairekodji ou «bœufs des rochers 》 peulhs de Bandiagara, Doventza, exportables vers le Ghana.

- les Sénokodji. Boufs peulhs de la plaine du Gondo-Séno. Exportables vers le Ghana.

La valeur relative de ces bœufs va croissant des Tiapatidji. (20 p. 100 de 'moins) jusqu'aux
Bourgoukodji, puis décroissant jusqu'aux Sénokodji ( $10 \%$ de moins environ):

La Physionomie du marché est donnée par la valeur du bœuf de tête, Ainsi connaissant la valeur du meilleur bouf Bourgou on peut non seulement avoir une idée de la valeur des diverses catégories de bovins mais aussi apprécier la variation des cours. En somme la valeur du bœuf étalon nous sert de référence de même que la valeur du kilogramme vif en Europe.

Le Bourgoukodji étalon mesure $1 \mathrm{~m}, 25$, pèse de 400 à $420 \mathrm{~kg}$; son rendement en carcasse est de l'ordre de $55 \%$.

Les boufs d'exportation pèsent de 320 à $420 \mathrm{~kg}$; leur rendement en carcasse varie de $48 \%$ à $55 \%$ (des rendements exceptionnels de $57 \%$ ont été constatés). Ils sont âgés de 6 ans au moins et de 12 ans au plus.

Les bœufs d'embouche et boufs destinés au travail sont des bœufs âgés de 3 à 5 ans. Certains sont achetés pour l'embouche et la préparation à l'exportation. D'autres, ceux de 3 à 4 ans, sont destinés au dressage pour le labour : cette catégorie se présente de plus'en plus nombreuse sur les marchés, car la demande pour le travail augmente. C'est pourquoi la valeur du $\mathrm{Kg}$ de cette catégorie de bétcil tend à rejoindre la valeur du $\mathrm{Kg}$ vif $\mathrm{du}$ bouf d'exportation (il y a quelques années cette valeur était de $20 \%$ moindre). Le bœuf de 4 ans apte au labour pèse de 260 à $280 \mathrm{~kg}$.

Les faurillons âgés de 1 c̀ 2 ans se présentent régulièrement sur le marché. La demande est soutenve par le besoin de placer l'argent, ainsi que de renouveler les attelages; le kg vif de cette catégorie de bétail est de 15 à $20 \%$ inférieur à la valeur du $\mathrm{kg}$ vif du bœuf d'exportation.'

Les veaux. Aucun veau n'est mis en vente car les vaches zébus ne restent en lactation qu'autant qu'elles sont suitées : il n'esł pas question pour. l'éleveur de tarir une vache en lui supprimant son veau .

Les bœufs de travail réformés sont les bœufsporteur (N'Gari-N'Daldi) et de plus en plus les bœufs de labour (N'Gari N'Domori) ayant fini leur temps, c'est-à-dire ayant passé l'âge de 10 ans au moins. C'est boufs ont souvent la corpulence des bœufs d'exportation; souvient même leur état d'embonpoint est-il meilleur ; cependant leur valeur n'atteint pas les $2 / 3$ de ces der- 
niers car ils sont inaptes à l'exportation, non pas que leur viande soit dépréciée, mais ils ne sont plus habitués à effectuer journellement les $30 \mathrm{~km}$ requis tout en s'alimentant. En effet, au cours de sa carrière, ce bétail a été habitué à être alimenté à l'attache par les fourrages ef les tourteaux qui lui sont distribués. Son éducation pour l'exportation ef l'incorporation aux Tiogal est à refaire ef c'est bien tard ; certains marchands l'entreprennent et réussissent cependant dans une honorable proportion.

Vaches stériles: Les «Rimaré ». La vache zébu, fertile dès 2 à 3 ans, produit en moyenne tous les deux ans. II n'est pas rare que trois ans se passent sans qu'elle soit fécondée: aussi bien la vache zébu n'est-elle convaincue de stérilité que passé l'âge de 6 ans. D'autre part ces vaches souvent conformées en bœuf et très appréciées pour l'exportation, sont préparées ef conservées le temps nécessaire à cette fin. Elles fournissent 2 à $3 \%$ des Tiogal, pèsent de 300 à $350 \mathrm{~kg}$ et valent aulant qu'un bouf d'exportation de même poids.

Vaches accidentellement inaptes à lo reproduction. Les tiques provoquent l'abcédation des trayons ce qui entraîne des mammites puis la perte d'un quartier, puis d'un autre, et enfin de la mamelle entière. Ce processus est d'autant plus fréquent et rapide selon que la région parcourve par le troupeau est plus infestée d'ixodes ou bien que les soins de détiquage sont moins bien exécutés par les bergers (dans certains troupeaux $30 \%$ des vaches ont perdu un quartier, $20 \%$ deux quartiers, $10 \%$ trois quartiers, $5 \%$ la mamelle entière). Ces vaches sans mamelles sont vendues car leur produit ne saurait être élevé : en général elles ne sont pas d'une qualité suffisante pour être exportées. Leur valeur dépend de leur état d'embonpoint (très variable).

- Vaches réformées. Les vieilles vaches (Raniawé) ne pouvant plus avoir de veaux se présentent relativement nombreuses sur les marchés et trouvent difficilement acquéreur, la demande des bouchers locaux étant très insuffisante ; cependant certains marchands Maures achètent les meilleures pour une destination assez mystérieuse. Ces vaches se cachectisent de marchés en marchés ; nombreuses sont celles qui finissent par mourir de leur belle mort. En moyenne le kilo- gramme de poids vif de ce bétail n'atteint pas le tiers de la valeur de celui du bœuf d'exportation.

Génisses. Les Nialé sont les génisses de 1 à 2 ans les Wigé sont les génisses pleines de 2 à 3 ans. Leur valeur est relativement considérable, la demande étant toujours supéricure à l'offre ; le kilogramme vif de Wigé peut valoir le double de celui du bœuf d'exportation et cela parce que la Wigé va bientôt devenir Naggé : la vache fertile que l'on doit conserver comme la poule aux œufs d'or.

\section{Les cours.}

Durant les dernières dix années les cours ont été stables, à l'image de l'élevage qui l'est aussi depuis que les grandes épizooties sont jugulées.

Le ucuuf de têle acheté pour l'exporlation valait en 1939 huit cents francs ; en 1949 il valait 12.000 francs et it vaut acfuellement 22.000 francs Aucune plus-value appréciable n'est constatée, compte-tenu des dévaluations du franc. Actuellement au Ghana le bœuf de tête se vend entre 30.000 et 45.000 francs suivant les fluctuations du marché soumis surtout aux: importantes variations de l'offre.

Pour situer la chose nous pouvons comparer le cours de notre bœuf à celui du bétail en France qui vient de subir une forte baisse due à la sécheresse.

Au Bourgou, le bœuf zébu de $400 \mathrm{~kg}$ vaut de 40 à 45.000 francs métro. En Normandie, le bœuf de $600 \mathrm{~kg}$ vaut 100.000 francs. En Vendée il se trouve à 70.000 francs.

Le kilogramme vif de bœuf Bourgou se commercialise donc entre 100 et 110 francs à la production. Le kilogramme vif de bétail métropolitain se commercialise lui, entre 120 et 170.

En admettant que les rendements soient semblables il faut reconnaître cependant que la qualité de la viande zébu est moindre; on ne peut donc pas dire que le bœuf d'exportation du Bourgou soit mal vendu.

Ces considérations étant faites voici quels sont les derniers cours enregistrés à Tenenkou ; ils sont de quelques points inférieurs à ceux dé Mopti. (en francs CFA). 
Taurillon

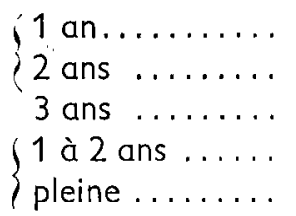

Taureau

3.000

6.000

10.000

Génisse

$\{$ pleine ..........

Vache stérile exportable .........

Vache réformée $\left\{\begin{array}{l}\text { pour inaptitude. } \\ \text { pour vieillesse. }\end{array}\right.$

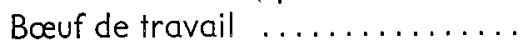

Bœuf $\quad\left\{\begin{array}{l}3 \text { ans } \ldots \ldots . . \\ 4 \text { d̀ } 6 \text { ans } \ldots . .\end{array}\right.$

Bœuf d'exportation.............

10 à 18.000

18 à 25.000

12.000

8.000

4.000

4 à 8.000

12.000

13 d̀ 16.000

16 à 22.000

L'importance quantitative des marchés Diawambé.

Les dénombrements qui ont pu être effectués nous permettent d'avancer les chiffres suivants :

Marchés de la rive gauche:

- Toguère, Kombe, Bovins présents annuellement ................

- Ovalo..........................

- Tenenkou

Marchés sur la rive droite: Les marchés de N'Gouma, Korientze, Kona, Fatoma, Mopti sont sensiblement d'égale valeur; le nombre des bovins qui s'y présentent annuellement est de 60.000 .

Au total les marchés Diawambé sont annuellement fréquentés par 80.000 bovins.

La signification de ce total brut doit être précisée en indiquant dans quelle proportion chacune des catégories de bovins le compose et d'autre part quel est le pourcentage d'animaux réellement commercialisés.

Les proportions sont quelque peu différentes dans les deux chaînes de marchés, ce que le contexte géographique explique, les marchés de la rive gauche se trouvant au sein de l'élevage et des cultures, ceux de la rive droite débouchant sur les pays importateurs de viande. Ainsi il peut être noté :

Marchés Marchés Rive gauche Rive droite

$\begin{array}{lll}\text { Bœufs d'exportation ..... } & 20 \% & 35 \% \\ \text { Bœufs de } 3 \text { à } 5 \text { ans . ..... } & 20 \% & 20 \% \\ \text { Vaches et bœufs réformés . } & 20 \% & 20 \% \\ \text { Taurillons et génisses..... } & 40 \% & 25 \%\end{array}$

Nous avons d'autre part indiqué que le total

brut des présentés ne reflète pas le nombre d'animaux réellement commercialisés, puisque un même animal peut être présenté plusieurs fois sur plusieurs marchés avant d'être vendu et que chaque fois il est décompté par nos agents. II devient donc nécessaire d'évaluer le pourcentage des animaux réellement commercialisés. En gros il est de $50 \%$ mais il y a lieu de préciser qu'il est différent suivant les catégories de bovins, ainsi nous estimons que $30 \%$ seulement des vaches et bœufs réformés sont effectivement commercialisés, et que les bœufs d'exportation le sont à $60 \%$

Nous pouvons donc avancer les chiffres suivants comptabilisant le nombre d'animaux vendus annuellement 'sur les marchés Diawambé :

$\begin{array}{lr}\text { Bœufs d'exportation ......... } & 15.000 \\ \text { Bœufs de } 3 \text { à } 5 \text { ans......... } & 8.000 \\ \text { Vaches et bœufs réformés .... } & 5.000 \\ \text { Taurillons et génisses....... } & \frac{12.000}{40.000}\end{array}$

Par cette étude des marchés, nous disposons dès lors d'éléments précieux pour évaluer le degré d'exportation du bétail (a).

Pour ce faire il nous faut connaître l'importance du troupeau exploité par les marchés Diawambé ainsi que sa composition.

Les marchés Diawambé exploitent : le cercle de Mopti ef une partie des cercles de Macina, de Niafunke, de Bandiagara dont le cheptel a pu être estimé assez précisément par les agents du Service de l'Elevage en tenant compte surtout du nombre des vaccinations effectuées. Avec le sentiment de ne pas nous écarter beaucoup de la vérité nous pouvons donc écrire que ces marchés exploitent un troupeau de 500.000 têtes environ.

Des recensements précis et détaillés effectués depuis plusieurs années sur des troupeaux complets formant un groupe de 8.000 têtes nous permettent de préciser la composition de ce troupeau :

(a) Pour cette évaluation, une méthode à rebours est classiquement employée, basée sur les statistiques issues du contrôle des exportations et des abattages, mais il est bien évident qu'ici la densité de notre réseau de surveillance, qui ne saurdit d'ailleurs être plus important, est insuffisante pour's'opposer aux fuites qui concernent surtout l'abattage. 


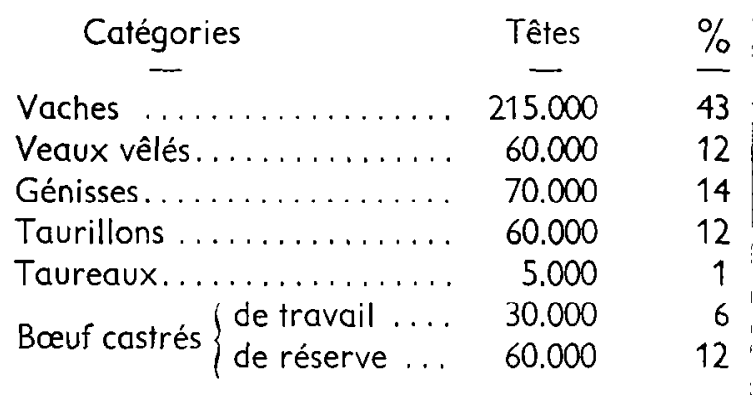

Nous laisserons de côté les renseignements que ces chiffres peuvent nous donner quant au croît du troupeau, à la fertilité des femelles, aux pertes subies par chaque génération jusqu'à l'âge adulte - pertes dues aux maladies, aux accidents de nutrition, aux fauves, aux accidents de transhumance, aux serpents - qui vont de 20 à $30 \%$. Nous nous bornerons à examiner l'exploitation du troupeau pour la viande, donc l'exploitation des bœufs de réserve, des bœufs de travail et des vaches qui devraient avoir, pour destinée finale, l'abattoir.

La réserve des bœufs costrés âgés de plus de 3 ans compte 60.000 têtes; annuellement, 15.000 têtes sont prélevées dans ce groupe pour l'exportation. Or nous avons vu qu'un bœuf n'est mûr pour cette destination qu'après avoir atteint l'âge de 6 ans, il nous faut donc constater que la totalité de cette fraction du troupeau est exploitée, puisque le quart de sa qualité est commercialisé annuellement ce qui veut dire que le stage dans la catégorie est en moyenne de 4 ans et que par conséquent 7 ans est l'âge moyen des bœufs évacués: l'exploitation des bœufs de réserve est donc dans l'état actuel des choses aussi porfaite que possible.

Les voches et les bœufs de travail peuvent être confondus lorsqu'il s'agit d'examiner le degré de leur exploitation pour la viande. En effet, leur réforme n'est décidée que lorsque passé dix ans toutes leurs ressources ont été épuisées : ils ont alors à peu près la même valeur commerciale. Ainsi 215.000 vaches et plus de 30.000 bœufs de travail âgés de 4 à 12 ans constituent cette partie du troupeau ( 5.000 d'entre eux sont commercialisés annuellement, soit $2 \%$ ). Admettons que seuls ceux d'entre eux ayant dépassé 10 ans doivent être exploités pour la viande : de cet âge il doit bien traîner 25.000 têtes. 5.000 d'entre elles sont commercialisées et 20.000 sont pratiquement perdues pour le consommateur au béné- fice des charognards et des hyènes. C'est évidemment là que quelque chose devrait être fait ; nous y reviendrons plus loin.

\section{II. - LES MARCHÉS BAMBARA}

L'examen des marchés Diawambé vient de nous donner des renseignements assez précis sur la commercialisation du bétail dans ta région du Delta Central du Niger. Il serait intéressant d'examiner de la même façon le marché du bétail dans la deuxième zone figurée sur notre carte, mais pour les raisons déjà indiquées, il ne faut pas compter serrer la question d'assez près pour pouvoir en tirer des conclusions valables : en effet les transactions sur le bétail se font dans cette zone d'une façon sporadique. II existe bien quelques marchés, mais les échanges entre vendeurs et acheteurs se font principalement à l'occasion et en tous lieux ; tout contrôle dans ces conditions devient donc pratiquement impossible.

Cependant nous devons noter ce que nous savons au sujet des marchés et du commerce du gros bétail dans cette zone.

Tout d'abord il faut signaler que dans cette région la fourniture de bœufs de travail aux cultivateurs est le but le plus important des transactions commerciales, l'exploitation du cheptel pour l'exportation passant au second plan.

Dans la zone considérée les marchés à bétail sont indépendants les uns des autres, squf ceux de Sokolo et Niono qui sont en pool.

Ces marchés ne sont pas organisés par des Diawambé ef l'on n'y rencontre pas les courtiers que sont les Teyfado, contrôleurs des marchés du Bourgou.

Les acheteurs sont surtout les agriculteurs. Rien n'est à ajouter à ce que nous en avons dit plus haut, de même pour les vendeurs qui sont les éleveurs de la région: Peulhs, Maures et agriculteurs.

Les animaux présentés sur ces marchés peuvent être classés de la même façon qué ceux des marchés Diowambé. Leurs origines ne sont pas les mêmes : nous y trouvons seulement des Tiapatidji (Maures), des Bouri, quelques Bourgoukodji et de plus des zébus peulhs de Ségou (type Toronké).

Nous pouvons noter la faible importance quantitative de la plupart des marchés connus dans 
cette zone; hormis le marché de Niono, leur importance est insuffisante pour qu'il soit intéressant d'entrer dans le détail.

Dans le cercle de Ségou nous connaissons les marchés de Konobougou, Baroueli, Souba, N'Gassola, Yolo, Dioro et celui de Segou, le plus important car il ravitaille les bouchers de la place : annuellement it se présente sur l'ensemble de ces marchés environ 10.000 têtes.

Dans le cercle de Macina le marché de Monimpebougou, qui ne fait pas partie des marchés Diawambé, présente annuellement environ 3.000 bovins ef en commercialise $75 \%$ qui sont achetés surtout par les colons de l'Office du Niger et les Maures.

Dans la subdivision de Niono deux marchés attachés au développement de l'ag riculture locale prennent de plus'en plus d'importance : Sokolo et Niono (le dimanche).

La marché de Sokolo présentera au total en 1959 environ 3.000 bovins; les animaux qui y sont invendus sont souvent dirigés sur Niono.

Le marché de Niono se développe depuis quelques années; il présentera en 1959 environ 10.000 bovins. Des statistiques effectuées sur plusieurs mois nous permettent d'indiquer les proportions de chacune des catégories de bovins présentés; elles donnent la physionomie du commerce du bétail dans la région, commerce lié surtout aux exigences de l'agriculture.

\begin{tabular}{|c|c|}
\hline $\begin{array}{l}\text { Veaux sevrés (1) } \ldots \\
\text { Taurillons........ } \\
\text { Jeunes taureaux. . . }\end{array}$ & $\left.\begin{array}{r}4 \% \\
24 \% \\
5 \%\end{array}\right\} 80 \%$ commercia- \\
\hline Génisses & $15 \%\left\{\begin{array}{l}80 \% \text { commercia- } \\
\text { lisés soit } 1.400\end{array}\right.$ \\
\hline $\begin{array}{l}\text { Vaches réformées... } \\
\text { Boufs réformés..... }\end{array}$ & $\left.\begin{array}{l}20 \% \\
20 \%\end{array}\right\} \begin{array}{r}40 \% \text { commercia- } \\
\text { lisés soit } 1.600\end{array}$ \\
\hline Vaches d'exportation & $2 \%, 80 \%$ commercia- \\
\hline Bœufs d'exportation. & $10 \%\{$ lisés soit 960 \\
\hline
\end{tabular}

Connaissant mal la valeur numérique du cheptel exploité par le marché de Niono nous ne tirerons pas, avec ces chiffres, de conclusions au sujet de l'exploitation du bétail comme nous l'avons fait après l'étude des marchés Diawambé mais nous sommes certains que les bovins mâles sont exploités au maximum pour le travail, ef que les femelles ef les boufs de trait sont très mal exploités pour la'viande. 'll én est de même dans toute cette zone comprenant le cercle de Ségou, la subdivision de Niono et la partie Ouest du cercle de Macina, que l'ón estime peuplée de 200.000 bovins fournissant annuellement 6 à 10.000 bœufs de travail, 4.000 bœufs d'exploitation et 3.000 bovins environ pour la boucherie locale.

De cet exposé nous pouvons retirer une conclusion d'importance : la vocation ancienne de l'élevage dans la région considérée était la production de boufs d'exportation. Aujourd'hui la production du bœuf de trait devient une activité concurrente, au détriment de la production de la viande (ce qui pourrait être évité). On n'omettra pas de tenir compte de cette évolution de l'exploi. tation du bétail dans la dernière partie de cette étude que nous abordons et où nous allons traiter des améliorations qui pourraient être apportées à l'exploitation du bétail.

\section{III. - CONSIDÉRATION SUR L'EXPLOITA- TION DU BÉTAIL ET LES AMÉLIORA- TIONS QUI POURRAIENT $Y$ ÊTRE AP- PORTÉES.}

Nous laisserons ici de côté l'exploitation des femelles pour la reproduction, le lait et le travail, ce qui nécessiterait une étude que nous n'avons pas abordée. 'Nous n'insisterons pas non plus sur l'exploitation du bétail pour le travail considérant que par la force même des chosès elle se développe normalement.

Nous allons nous borner à examiner l'exploitation du bétail pour la viande, persuadés qu'en plus des améliorations déjà effectuées, quelque chose pourrait être entrepris dans 'ce domaine pour obtenir un meilleur rendement du troupeau

La production du bœuf d'exportation capable de se rendre à pied en Basse-Côte nous semble parfaite. Le modèle réalisé possède les qualités indispensables au but proposé : seule, sa précocité pourrait faire l'objet de quelques critiques, mais encore ne faut-il pas confondre rapidité de croissance et rapidité d'entraînement à la marche. Dans l'esprit de l'éleveur européen le mot n'est pas attaché à cette préparation spéciale et pourtant c'est là seulement que du temps pourrait peul-être être gagné. En effet, il n'est pas question de chercher à accélérer la croissance du zébu peuth par l'introduction d'un sang européen : que ferions-nous à Missibougou par exemple, 
d'un métis bien venu mais incapable de s'exporter lui-même. Abattons-le sur place, dironsnous, réfrigérons-le et envoyons par avion cette parfaite carcasse à Abidjan ou Accra... D'accord, mais il ne s'agit pas de cela; il s'agit d'abord d'apporter de la viande sur place dans les villages pour longtemps encore dépourvus de chambres froides, et difficilement accessibles par la route. Seuls nos zébus peulhs en sont capables à l'heure actuelle, et pour longtemps encore. II faut donc continuer à les produire tels qu'ils sont, avec de simples améliorations de nutrition et de condition de vie.

La commercialisation de ce bétail telle qu'elle est effectuée par les Diawombé nous semble aussi très peu perfectible, l'organisation que ces marchands ont mise sur pied étant difficile à dépasser. Les pertes numériques que subissent les tiogals sont infimes ( $1 \%$ au maximum) ; cependant les pertes de poids dues aux trypanosomiase contractées dans le sud sont sensibles. On peut y remédier par une chimio-prévention spécifique ; certains utilisent cette méthode, d'autres pas ; elle devrait être obligatoire ef même gratuite au départ.

Ainsi l'exploitation des bøufs classiquement préparés pour l'exportation nous semble difficile à améliorer et de ce fait toujours valable ; il n'en est pas de même pour les autres catégories de bovins.

Les bøufs d'embouche de 3 à 5 ans doivent attendre la fin de leur entrainement pour être exportés mais un fait nouveau va intervenir : le développement des routes et du camionnage. Il est bien évident que le transport par camion permet l'expédition de jeunes boufs encore mauvais marcheurs; cela n'a pas échappé a certains marchands favorablement placés à proximité de bonnes routes. Ainsi dernièrement une centaine de bœufs sont partis de Niono et de Ségou pour Bouaké. Le prix du transport est de 4 à 5.000 francs par tête : l'opération est donc très rentable.

Lorsque l'état des routes permettra des transports moins onéreux, cette façon d'exploiter le troupeau sera recommandable et présentera d'autre part l'avantage certain de décharger les pâturages.

Par ce moyen aussi les boufs et vaches réformés encore en bon état pourraient être convenablement exploités mais seraient-ils assez résis- tants pour, du lieu de débarquement, se rendre au village consommateur? Nous pouvons proposer mieux pour l'exploitation de cette catégorie de bovins,

L'étude des marchés Diawambé nous a fait apparaître la déplorable médiocrité de l'exploitation des vaches et des boufs de trait pour la viande. A l'appui de cette constatation il estbon d'examiner ici le cas cu troupeau des colons de l'Office du Niger, organisme qui pourrait être intéressé à la solution du problème.

Les paysans de l'O. $N$. sont, comme tous autres paysans venus à la culture attelée, placés désormais devant le problème de l'élevage, problème qui, de par leurs origines ne leur était pas familier, mais qu'ils sont amenés, vaille que vaille, à résoudre. Ils ont appris à utiliser le boeuf de trait, à l'acheter, à l'entretenir ; ils apprennent à le produire et en entreprennent l'élevage. Ce bétail est étroitement surveillé ef recensé par les infirmiers vétérinaires de l'O. N., aussi (mis à part les veaux) les chiffres dont nous disposons forment assez exactement le spectre des troupeaux dont disposent actuellement de telles collectivités adonnées à la culture attelée.

Actuellement le troupeau des colons de l'Office du Niger (hormis Baguineda) est cinsi composé :

\begin{tabular}{|c|c|c|}
\hline Bceufs & $53.3 \%$ & 10.155 \\
\hline Vaches & $19,1 \%$ & 3.650 \\
\hline Génisses. & $13,4 \%$ & 2.554 \\
\hline Taurillons & $12,8 \%$ & 2.446 \\
\hline Veau... & $1,4 \%$ & 416 \\
\hline & $100 \%$ & 9.060 \\
\hline
\end{tabular}

L'exploitation de ce troupeau pour le travail est bonne. Par contre son exploitation pour la viande est à peine envisagée. D'après les statistiques dont nous disposons nous prévoyons que moins de 200 bœufs et vaches réformés seront vendus ou abattus au cours de l'année présente : soit moins de $1,5 \%$ des adultes:

Les colons de l'Office disposent d'environ 14.000 bovins adultes de tous âges. Une bonne économie voudrait donc que 2.000 anciens soient éliminés chaque année. Mais comment? II n'y a pas actuellement de débouchés suffisants pour cette catégorie de bovins; à part quelques-uns vendus à temps avant la décrépitude par des propriétaires mieux avisés, la plupart «dis- 
paraissent en fumée » après avoir inutilement usé les pâturages au détriment des jeunes.

II s'agit donc de trouver un débouché lucratifpour les vieux animaux : nous pensons le trouver dans la conserverie. Le marché peut s'ouvrir: une boîte de bœuf peut arriver dans la case la plus reculée ; cette boîte peut, comme la boîte de sardine, être ouvertesur n'importe quel bord de route.

D'ailleurs cela n'est plus une idée originale : la preuve en est que nous avons en main une boîte de conserve d'une livre achetée $120 \mathrm{fr}$ dans une boutique de Niafunké, fabriquée au Nigeria, à Kano, et contenant du riz, du bœuf, du poivre, de la tomate, des oignons, etc... Personne ne peut douter que l'Office du Niger soit désormias bien placé à Markala pour suivre cet exemple.

\section{CONCLUSION}

Ainsi l'éfude du'marché du gros bétail au Moyen-Soudan nous fait apparaître les points suivants :

La production ef l'exploitation des boeufs d'exportation est une bonne affaire bien menée telle qu'elle l'est, seule capable à l'heure actuelle, de fournir de la viande aux agglomérations lointaines de la Basse-Côte, agglomérations non pourvues des installations nécessaires à l'utilisation des viandes réfrigérées.

L'exploitation des bœufs encore trop jeunes pour s'exporter eux-mêmes, se développe dans la mesure où l'état des routes permet un camionnage plus facile.

L'exploitation du troupeau pour le travait va en s'amplifiant ef dans l'état actuel des choses au détriment de la production de la viande.

L'exploitation des bovins ayant terminé leur temps utile de reproducteurs et de travailleurs est d'une déplorable médiocrité, faute d'avoir trouvé un moyen de les utiliser; ces bovins encombrent les troupeaux, usent les pâturages au détriment des jeunes. II est donc de toute nécessité de les éliminer. Nous proposons pour que cette élimination soit lucrative de lancer l'industrie de la conserve, grâce à laquelle les villages les plus isolés pourraient être mieux alimentés en protéines.

\section{SUMMARY \\ The Marketing of Cattle in Lower Soudan (Mali).}

The region in the bend of the Niger River, is favourable geographically and economically to the establishment of large markets which have the objectives of providing meat to the interior, supplying draught animals to cultivators, and the export of slaughter stock to areas towards and at the coast. The authors describe the present system and those who are involved and the types of cattle which pass through these markets. Their studies show that while the export trade is fairly well organised, and the movement of animals too young to stand the road journey, could be effected by lorry along improved stock routes, the culling of the animals for local consumption and as draught oxen is deplorable. The authors are of the opinion that these animals could be eliminated in a more profitable manner, by the establishment of a cannery.

\section{RESUMEN}

\section{El mercado de ganado mayor en Sudán-medio.}

La región de la delta del Niger es, geográfica y económicamente favorable a la existencia de importantes mercados de ganado con una triple finalidad : proporcionar carne al mercado interior, suministrar bueyes de trabajo a los agricultores y exportar animales de carnicería hacia los países de las costa.

Los autores hacen una exposiciôn de la organizacion de estos mercados y de las personas que los frecuentan (vendedores, compradores, carniceros, exportadores, corredores), y describen el ganado que alli se presenta.

El estudio de estos mercados muestra, que si bien la producción y explotación de bueyes con destino al mercado de fuera son bien llevados, la explotación de bueyes todavía muy jóvenes para hacer el traslado caminando hasta las zona de consumo debe desarrollarse convenientemente o bien mejorar los medios de comunicación que permitan su traslado en camiones. La explotación para carnes de vacas y bueyes muy deformes es deplorables. Los autores piensan que sería posible eliminar de una manera económica estos animales mediante su industrialización. 